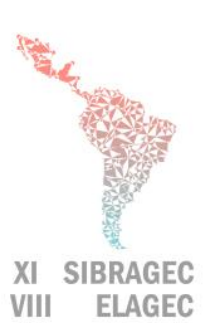

VIII ELAGEC

\section{SIMPÓSIO BRASILEIRO DE GESTÃO E ECONOMIA DA CONSTRUÇÃO}

VIII ENCUENTRO LATINOAMERICANO DE GESTIÓN Y ECONOMÍA DE LA CONSTRUCCIÓN

Do conhecimento à ação: práticas avançadas de gestão da produção Londrina, Paraná, Brasil. 23 a 25 de Outubro de 2019

\title{
ANÁLISE DE TEMPOS PRODUTIVOS E IMPRODUTIVOS EM UMA OBRA HORIZONTAL POR MEIO DE SIMULAÇÃO COMPUTACIONAL
}

\author{
YAMAMOTO, Lucio S. (1); VIVAN, André L. (2); SERRA, Sheyla M.B. (3) \\ (1) Universidade Federal de São Carlos, (16) 3351-9670, lucioyamamoto@ufscar.br, (2) Universidade \\ Federal de Itajubá, andrevivan@unifei.edu.br, (3) Universidade Federal de São Carlos, \\ sheylabs@ufscar.br
}

\begin{abstract}
One of the causes of waste in construction may be the failure to plan production processes in which activities that represent physical flows are not normally considered. Nevertheless, these are fundamental activities for the production process development and may represent the waiting of service, the displacement of workers and the materials transportation. According to the lean mindset, flow activities do not add value to the work product and should be optimized to reduce waste. Thus, by considering the principles of pull construction, the conversion activities for both services were studied and simulated: structural masonry and on-site slab using ProModel@ software. The real data of a project were obtained, and the computer simulation was performed for two planning scenarios. It was concluded that the methods related to pulled construction actually added efficiency and speed to the work, reducing delays and service inventories, since it was verified a reduction of $24,13 \%$ in the delivery of the activities when it was simulated without optimizations and $54,55 \%$ in the delivery time of the work with the optimizations proposed by the software.
\end{abstract}

Keywords: Construction, pulled construction, processes, waste of time, modeling.

\section{INTRODUÇÃo}

O sucesso da construção civil depende da concepção de cenários de planejamento da execução que considerem o correto funcionamento do canteiro de obras, normalmente realizada por equipes multidisciplinares. Diversas construtoras, no entanto, não definem formalmente os processos de planejamento, dificultando a interação entre as várias equipes de produção (VIVAN; PALIARI, 2012).

Sacks et al. (2010) mencionam que como existem vários intervenientes trabalhando simultaneamente durante a execução de uma obra, é comum observar canteiros de obra desorganizados e com excessos de fluxos de trabalho e de circulação, além da ocorrência de excesso de tempo de espera, retrabalhos, estoques inadequados de materiais, movimentação e manuseio desnecessário dos materiais, entre outros. Com isso, um dos grandes desafios na busca da eficiência de uma construção é conseguir um fluxo de trabalho contínuo, por meio de um planejamento apropriado da construção, a fim de obter a eficácia na gestão da produção (Bortolini et. al, 2014). 
A Construção Enxuta, com princípios de gerenciamento e controle proveniente da mentalidade enxuta, surge como uma nova filosofia de trabalho, na qual a gestão é focada na redução de vários tipos de desperdícios e consequente melhoria do planejamento e da produtividade dos operários, como indicado por Erol; Dikmen; Birgonul (2016) e Li et al. (2017).

Assim, este trabalho é referente a uma análise acerca da implementação de conceitos de construção puxada em um canteiro de obras buscando os melhores resultados possíveis quanto ao tempo de entrega do produto e à redução de estoques de serviços na construção de uma edificação horizontal, fazendo uso do Tempo Takt como uma das métricas principais de análise. $\mathrm{O}$ objetivo do trabalho é apresentar duas simulações de processos de trabalho a partir de modelagens num programa computacional com base num estudo real.

\section{CONCEITUAÇÃO}

- Produtividade: Teoricamente, produtividade é definida como uma relação entre as saídas da produção e as entradas que essa produz (YI; CHAN, 2013). De maneira mais específica, a medição da produtividade é uma expressão do resultado físico ou o volume real de bens e serviços, os quais podem ser definidos como as saídas da produção da construção civil, relacionada com a quantidade real e/ou física das entradas.

- Tempos improdutivos na construção civil: Tal conceito deve ser entendido como qualquer ineficiência que reflita no uso de equipamentos, materiais, capital e mão de obra em quantidades superiores àquelas previstas e/ou necessárias para a produção da edificação em si. Sendo assim, diversos autores como Yang et al. (2014) e Formoso et al. (1997) indicam que perdas englobam tanto o desperdício dos insumos necessário para a realização do produto final, como a intensa execução de tarefas adicionais que podem gerar custos extras e atividades que agregam valor.

Como representado na Figura 1, a construção civil apresenta diversas causas que impedem seu desenvolvimento. A melhoria do design, gestão e, principalmente, de fatores do local de trabalho e ambiente são essenciais na busca pela melhoria e norteadores para o desenvolvimento da construção enxuta no país.

\section{Figura 1 - Análise dos fatores de baixa produtividade}

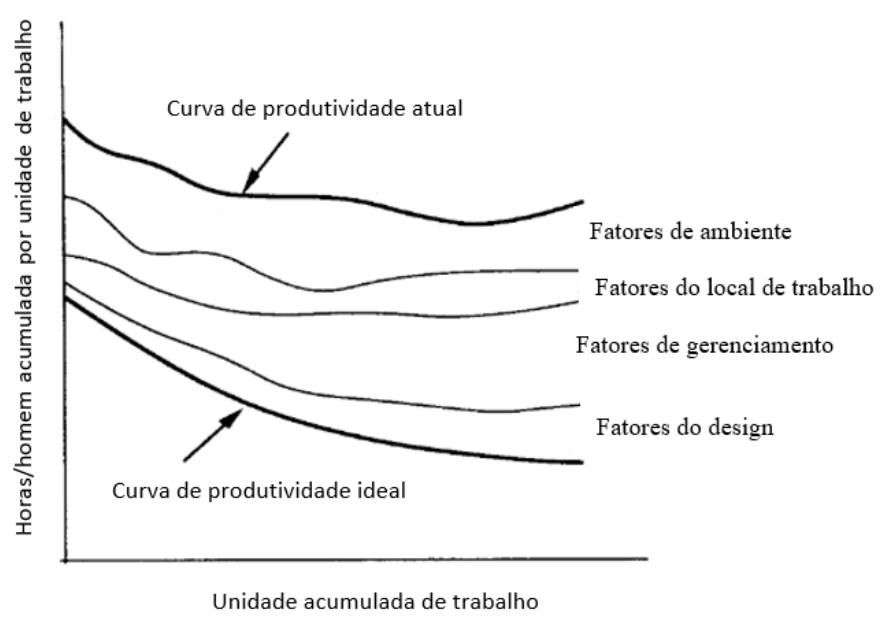

Fonte: Alarcón (1993) 
SIBRAGEC - ELAGEC 2019 - de 23 a 25 de Outubro - LONDRINA - PR

- Takt-time: O Takt-time é definido a partir da demanda do mercado e do tempo disponível para produção; é o ritmo de produção necessário para atender a demanda. Matematicamente, resulta da razão entre o tempo disponível para a produção e o número de unidades a serem produzidas (ALVAREZ; ANTUNES JR., 2001). Ainda é possível compreender que esse tempo é o designado para atender a produção em uma célula ou linha. A Equação 1 indica o modo de cálculo simples de tal ferramenta:

$$
\text { Takt }- \text { time }=\frac{\text { Tempo de produção disponível }}{\text { Demanda do cliente }}
$$

\section{MÈTODO DE PESQUISA}

O método de pesquisa utilizado foi o de estudo de caso seguido pela simulação computacional.

O estudo de caso consistiu inicialmente no levantamento de dados junto a um empreendimento localizado na cidade de Limeira - SP, constituído por 76 casas assobradadas e geminadas duas a duas, com $120 \mathrm{~m}^{2}$ cada em alvenaria estrutural de blocos de concreto. As unidades de produção (conjunto de duas casas geminadas) foram agrupadas em oito conjuntos de casas (com quatro ou cinco unidades de produção) que foram numeradas segundo a sequência de execução como ilustrado na Figura 2.

Figura 2 - Esquema de execução proposto no planejamento

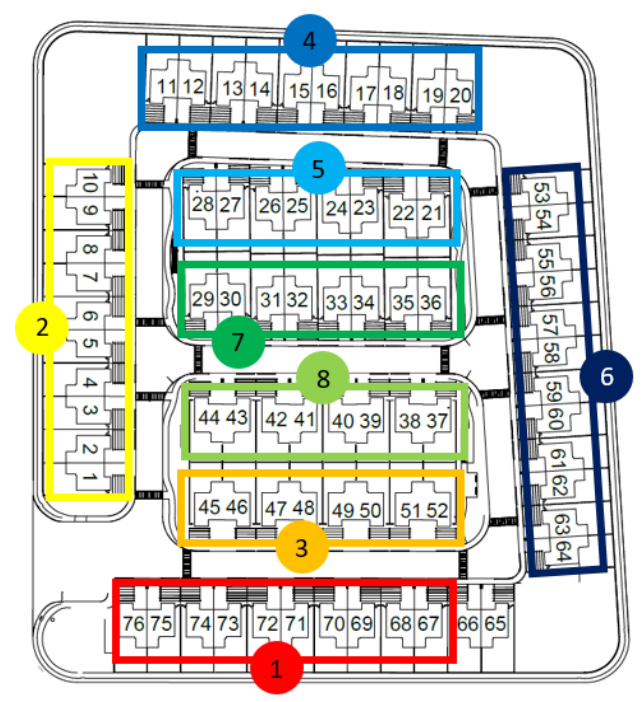

Os serviços estudados foram referentes à execução da alvenaria estrutural do pavimento térreo, sendo que essas eram compostas por 4 equipes com 4 colaboradores cada, e à execução da laje pré-moldada do pavimento térreo com 2 equipes com 2 colaboradores. Foi realizado o levantamento da produtividade destas equipes a fim de se determinar o seu indicador médio.

A simulação é considerada uma estratégia de pesquisa que visa simular o comportamento de um sistema durante um certo período, de maneira quantitativa, agindo sobre as variáveis e os parâmetros do modelo construído para representar este sistema (BANDEIRA, 2009). Assim, a Figura 2 indica o a numeração das unidades e conjuntos que serviram de base para a modelagem computacional. Os conjuntos numerados representam a sequência de execução da obra que também foi respeitada na modelagem computacional. 
Deve-se indicar que a modelagem foi realizada apenas frente às atividades de conversão estudadas (alvenaria estrutural e laje), sem a consideração de atividades de fluxo. $\mathrm{O}$ software ProModel ${ }^{\circledR}$ é uma ferramenta de simulação que permite a modelagem de todos os tipos de sistema, sendo que sua modelagem é baseada na orientação de objetos. Além disso, o software utiliza uma ferramenta de otimização chamada SimRunner que realiza análises "E se" pela execução de um planejamento prévio e fatorial de experimentos no modelo, possibilitando uma resposta extremamente próxima da realidade (BENSON, 1997). Neste recurso, os objetivos são pré-definidos e aplicados a locais, entidades, recursos, variáveis e custos. As restrições são elencadas pelo SimRunner de acordo com as variáveis atribuídas ao modelo (macros).

Foram realizadas duas diferentes simulações, sendo a primeira considerada "Não Otimizada" correspondente à reprodução das situações observadas em canteiro; e uma segunda modelagem "Otimizada" considerando a situação de equilíbrio entre as equipes de trabalho constantes do estudo.

Finalizando, os resultados obtidos com os dois modelos simulados foram comparados, gerando conclusões sobre a presente pesquisa.

\section{RESULTADOS E DISCUSSÕES}

\subsection{Análise entre a simulação real (não otimizada) e otimizada}

A Figura 3 ilustra a interface e utilização do software na qual estão presentes as indicações dos locais onde são executados os serviços, sendo, portanto, os locais de análise de produtividade dos operários.

\section{Figura 3 - Definição dos locais para modelagem}

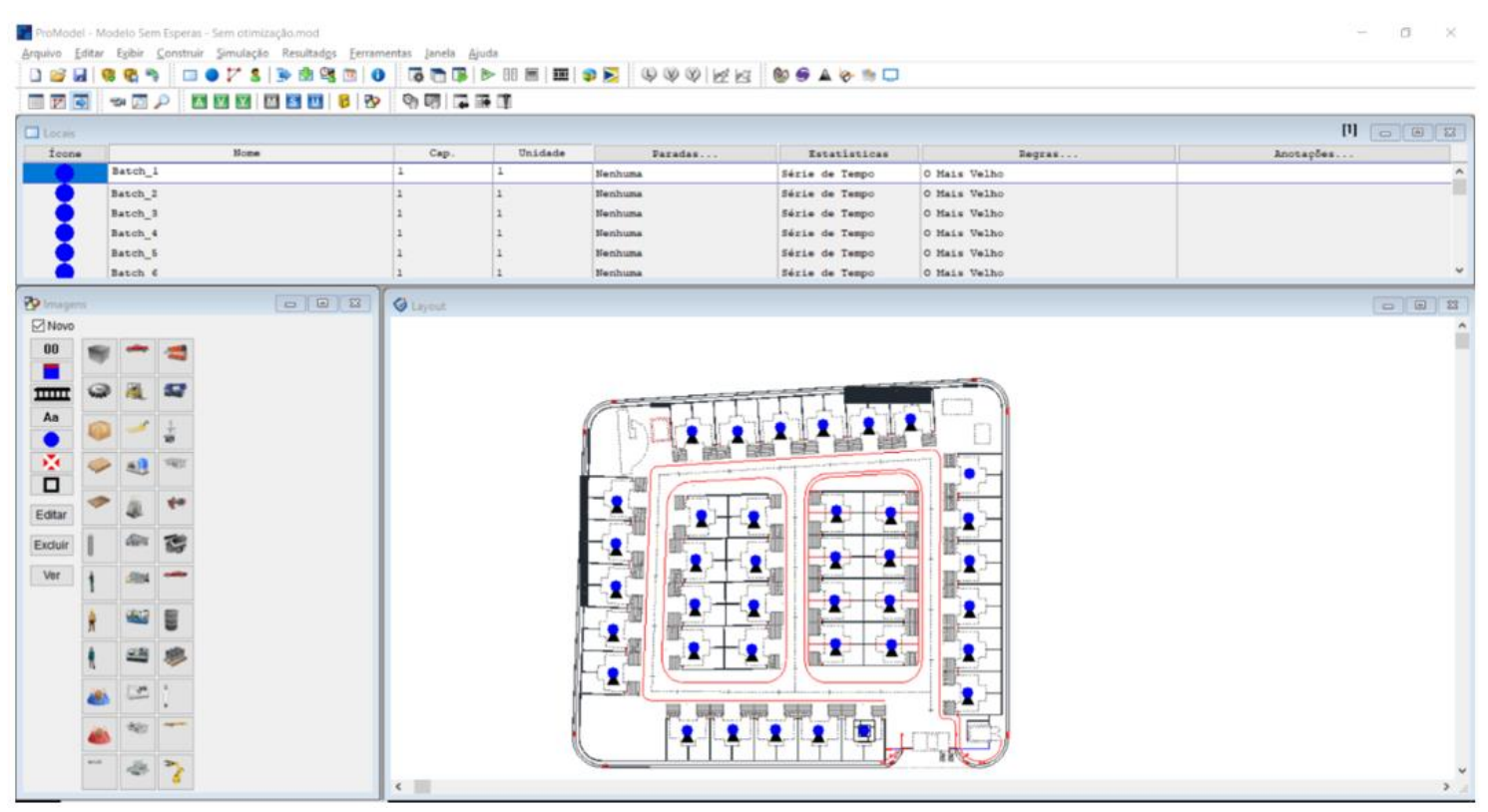

Fonte: Captura de tela do software ProModel® 
SIBRAGEC - ELAGEC 2019 - de 23 a 25 de Outubro - LONDRINA - PR

O Quadro 1 indica a diferença em relação às médias dos estados dos locais entre a simulação "Não Otimizada" e a simulação "Otimizada", sendo que a "Otimizada" possui o dobro de colaboradores da "Não otimizada" em cada equipe de alvenaria.

\section{Quadro 1 - Comparação dos estados dos recursos entre as simulações}

\begin{tabular}{|c|c|c|c|c|c|c|}
\hline \multirow{2}{*}{$\begin{array}{c}\text { Elemento } \\
\text { Equipes }\end{array}$} & \multicolumn{2}{|c|}{$\begin{array}{l}\text { Número de } \\
\text { colaboradores }\end{array}$} & \multicolumn{2}{|c|}{ \% Em Uso } & \multicolumn{2}{|c|}{ \% Ocioso } \\
\hline & $\begin{array}{c}\text { Não } \\
\text { otimizado }\end{array}$ & Otimizado & $\begin{array}{c}\text { Não } \\
\text { otimizado }\end{array}$ & Otimizado & $\begin{array}{c}\text { Não } \\
\text { otimizado }\end{array}$ & Otimizado \\
\hline $\begin{array}{c}\text { Equipe de } \\
\text { Alvenaria A }\end{array}$ & 4 & 8 & 89,42 & 72,70 & 10,58 & 27,30 \\
\hline $\begin{array}{c}\text { Equipe de } \\
\text { Alvenaria B }\end{array}$ & 4 & 8 & 83,21 & 67,65 & 16,79 & 32,35 \\
\hline $\begin{array}{c}\text { Equipe de } \\
\text { Alvenaria C }\end{array}$ & 4 & 8 & 91,9 & 74,72 & 8,1 & 25,28 \\
\hline $\begin{array}{c}\text { Equipe de } \\
\text { Alvenaria D }\end{array}$ & 4 & 8 & 89,42 & 72,70 & 10,58 & 27,30 \\
\hline \multicolumn{3}{|c|}{ Média } & 88,49 & 71,94 & 11,51 & 28,06 \\
\hline $\begin{array}{l}\text { Equipe de } \\
\text { Laje A }\end{array}$ & 2 & 2 & 49,68 & 80,78 & 50,32 & 19,22 \\
\hline $\begin{array}{l}\text { Equipe de } \\
\text { Laje B }\end{array}$ & 2 & 2 & 42,23 & 68,66 & 57,77 & 31,34 \\
\hline \multicolumn{3}{|c|}{ Média } & 45,96 & 74,72 & 54,05 & 25,28 \\
\hline
\end{tabular}

O Quadro 1 demonstra que a inclusão de novos colaboradores no serviço de alvenaria levou a uma redução nos tempos em uso, já que menos trabalho era disponibilizado na atividade em questão. No entanto, o dobro de colaboradores responsáveis pela alvenaria possibilitou substancial aumento no tempo em uso dos recursos de execução da laje.

Isso ocorreu em razão das atividades estão conectadas em relação a entregas no caso da simulação com princípios da construção puxada. Nesse caso, o serviço da laje é cliente do serviço da alvenaria, acarretando em um impacto direto nos tempos ociosos e de uso.

Os dados demonstram que, em média, houve um aumento do tempo em uso das equipes de laje de $28,76 \%$ compensando a queda média de $16,55 \%$ do tempo de uso das equipes de alvenaria. Tais dados influenciaram significativamente a diminuição do tempo de entrega das duas atividades juntas.

\subsection{Análise entre valores qualitativos dos cenários da obra}

Por fim, é possível comparar a produtividade e o Tempo Takt da obra real com as situações simuladas. O Quadro 2 apresenta os dados obtidos para cada situação de estudo.

O Tempo Takt diminui de $0,243 \mathrm{~h} / \mathrm{m}^{2}$ da obra real para $0,0775 \mathrm{~h} / \mathrm{m}^{2}$ na simulação "Não Otimizada" e, em seguida, para $0,0465 \mathrm{~h} / \mathrm{m}^{2}$ na simulação "Otimizada". Isso é um dado positivo, já que o Tempo Takt indica o ritmo da obra, fazendo uma analogia de seu significado original. Compreende-se que a obra está atingindo patamares mais eficazes, já que, por ser uma construção horizontal, o Tempo Takt também é o Tempo de Ciclo da obra. Por essa razão, sua correspondente redução implica em uma obra com ritmos mais acelerados. A comparação foi feita com o Takt das atividades como um todo por se tratar de uma linha que busca traçar um limite de ação de tempo das atividades. 
SIBRAGEC - ELAGEC 2019 - de 23 a 25 de Outubro - LONDRINA - PR

Quadro 2 - Comparação dos tempos das obras analisadas

\begin{tabular}{|l|c|c|c|}
\hline \multicolumn{4}{|c|}{ Comparação dos tempos das obras } \\
\hline \multicolumn{1}{|c|}{ Análise } & Obra real & $\begin{array}{c}\text { Simulação Não } \\
\text { Otimizada }\end{array}$ & Simulação Otimizada \\
\hline Produtividade - Laje $\left(\mathrm{m}^{2} / \mathrm{Hxh}\right)$ & 0,319 & 0,801 & 1,335 \\
\hline $\begin{array}{l}\text { Produtividade - Alvenaria } \\
\left(\mathrm{m}^{2} / \mathrm{Hxh}\right)\end{array}$ & 0,336 & 0,606 & 0,505 \\
\hline Tempo Takt $\left(\mathrm{h} / \mathrm{m}^{2}\right)$ & 0,243 & 0,0775 & 0,0465 \\
\hline
\end{tabular}

A comparação da produtividade da alvenaria demonstra que ela aumentou da obra real para a simulação "Não Otimizada", porém diminui da "Não Otimizada" para a "Otimizada". Os números tiveram essa tendência, pois na simulação "Otimizada" o número de oficiais dobrou para uma mesma metragem quadrada de serviço. No entanto, o tempo de execução não reduziu linearmente ao aumento dos oficiais, gerando uma diminuição na produtividade, com valor ainda maior que a da obra real.

Por fim, compara-se a produtividade das equipes da laje, a qual cresceu em todas as análises, passando de $0,319 \mathrm{~m}^{2} / \mathrm{Hh}$ para $0,801 \mathrm{~m}^{2} / \mathrm{Hh}$ e finalizando com $1,335 \mathrm{~m}^{2} / \mathrm{Hh}$. Essa comparação é fundamental para o entendimento do conceito e aplicação da construção enxuta como uma ferramenta de gestão e planejamento. Enquanto na obra real a execução da laje era realizada quando possível, a simulação "Não Otimizada" demonstrou que a produção por demanda de clientes é mais eficiente e efetiva, já que busca eliminar esperas entre atividades de execução. A simulação "Otimizada" ainda promove uma análise paralela do aumento da produtividade da execução da laje. Por se tratar de uma simulação puxada, mesmo aumentando o número de equipes da alvenaria, foi a produtividade da laje que se elevou, já que a laje é uma atividade cliente da alvenaria.

\section{CONSIDERAÇÕES FINAIS}

A construção puxada realmente apresenta melhorias frente aos métodos tradicionais de construção empurrada. Ainda assim, é importante apontar que os dados da simulação não preveem nenhum imprevisto construtivo e trabalha em perfeição de planejamento.

Destaca-se que o modelo de simulação pode incorporar determinadas variáveis com o intuito de se simular imprevistos e paradas não programadas, fato que depende da precisão do modelo frente às condições locais do canteiro de obras e dos objetivos de análise da simulação.

Os resultados demonstram que a implementação de técnicas da construção puxada promove, por si só, a redução do tempo de entrega e, consequentemente, o aumento da produtividade. A simulação foi realizada como maneira de provar computacionalmente a relação entre as atividades e seus tempos de ciclo, visando o tempo de entrega final do empreendimento.

Finalmente, entende-se que a pesquisa aqui apresentada pode ser complementada com a análise dos processos que compõem as atividades estudadas, buscando entender e otimizar as características físicas do canteiro de obra que possam colaborar na redução do tempo das atividades que não agregam valor à obra. 
SIBRAGEC - ELAGEC 2019 - de 23 a 25 de Outubro - LONDRINA - PR

\section{REFERÊNCIAS}

ALVAREZ, R. D. R.; ANTUNES JR., J. A. V. Takt-time: conceitos e contextualização dentro do Sistema Toyota de Produção. Gestão \& Produção, v.8, n.1, p.1-18, 2001.

ALARCÓN, L. F. Modeling waste and performance in construction. 20 p. Departamento de Gestão e Engenharia da Construção, Universidade Católica do Chile, Santiago, Chile, 1993.

BANDEIRA, M. Tipos de pesquisa. 2009, 10p. Disponível em:

http://paginapessoal.utfpr.edu.br/kathya/Disciplinas/introducao_engenharia/introducao-aengenharia/Simulacao.pdf. Acesso em julho de 2019.

BENSON, D. Simulation modeling and optimization using promodel. (D. H. W. S. Andradbttir, K. J. Healy, B. L. Nelson, Eds.) Orem, UT 84097, USA: PROMODEL

Corporation, 1997

BORTOLINI, R.; SOUZA, F.J.C.; FORMOSO, C.T. Uso integrado de simulação 4D com prática Lean em um empreendimento de um Hotel em Porto Alegre. In: $4^{\circ}$ Congresso Sistemas LEAN, 2014. Anais..., Porto Alegre - RS, p.236-250.

EROL, H.; DIKMEN, I.; BIRGONUL, M. T. Measuring the impact of lean construction practices on project duration and variability: A simulation-based study on residential buildings. Journal of Civil Engineering and Management, v.23, n.2, p.241-251, 2016.

FORMOSO, C.T.; CESARE, C.M.; LANTELME, E.M.V.; SOIBELMAN, L. As perdas na construção civil : conceitos, classificações e seu papel na melhoria do setor. 12 p. Porto Alegre, Rio Grande do Sul, UFRGS, 1997.

LI, S.; WU, X.; ZHOU, Y.; LIU, X. A study on the evaluation of implementation level of lean construction in two Chinese firms. Renewable and Sustainable Energy Reviews, v.71, p. 846$851,2017$.

SACKS, R.; DAVE, B.; KOSKELA, L.; OWEN, R.. Analysis framework for the interaction between lean construction and building information modelling, In: XVII Internacional Group Lean Construction (IGLC), 2010. Proceedings... Taiwan, China.

VIVAN, A.L.; PALIARI, J.C. Design for Assembly aplicado ao projeto de habitações em Light Steel Frame. Ambiente Construído, Porto Alegre, v.12, n.4, p.101-115, 2012.

YANG, J.B.; HUANG, K.M.; LEE, C.H., CHIU, C.T. Incorporating lost productivity calculation into delay analysis for construction projects. KSCE Journal of Civil Engineering, v.18, n.2, p.380-388, 2014.

YI, W.; CHAN, A.P. Critical review of labor productivity research in construction journals. Journal of Management in Engineering, v.30, n.2, p.214-255, 2013.

\section{AGRADECIMENTOS}

Agradecemos o apoio do CNPq por viabilizar o uso do software Promodel®. da CAPES pela bolsa de estudo, da FAPESP pela divulgação, assim como à empresa colaboradora com a pesquisa. 